\title{
NUMERICAL INVESTIGATION OF UNSTEADY FLOW PAST A CIRCULAR CYLINDER USING 2-D FINTTE VOLUME METHOD
} Md. Mahbubar Rahman', Md. Mashud Karim² and Md. Abdul Alim ${ }^{3}$

${ }^{1}$ Department of Natural Science, Stamford University Bangladesh, Dhaka-1209, ${ }^{2}$ Dept. of Naval Architecture and Marine Engineering, BUET, Dhaka-1000, ${ }^{3}$ Department of Mathematics, BUET, Dhaka-1000, Bangladesh.

E-mail: ${ }^{1}$ mahbub_stamford@yahoo.com, ${ }^{2}$ mmkarim@name.buet.ac.bd, ${ }^{3}$ maalim@math.buet.ac.bd

\begin{abstract}
The dynamic characteristics of the pressure and velocity fields of unsteady incompressible laminar and turbulent wakes behind a circular cylinder are investigated numerically and analyzed physically. The governing equations, written in the velocity pressure formulation are solved using 2-D finite volume method. The initial mechanism for vortex shedding is demonstrated and unsteady body forces are evaluated. The turbulent flow for Re $=1000$ \& 3900 are simulated using $k-\varepsilon$ standard, $k-\varepsilon$ Realizable and $k-\omega$ SST turbulence models. The capabilities of these turbulence models to compute lift and drag coefficients are also verified. The frequencies of the drag and lift oscillations obtained theoretically agree well with the experimental results. The pressure and drag coefficients for different Reynolds numbers were also computed and compared with experimental and other numerical results. Due to faster convergence, 2-D finite volume method is found very much prospective for turbulent flow as well as laminar flow.
\end{abstract}

Keywords: Viscous unsteady flow, laminar \& turbulent flow, finite volume method, circular cylinder.

$\begin{array}{ll}\text { NOMENCLATURE: } \\ a & \text { Radius of the circular cylinder } \\ \mathrm{D} & \text { Diameter of the circular cylinder } \\ \rho & \text { Density of the fluid } \\ \mu & \text { Coefficient of viscosity } \\ \mathrm{t} & \text { Time } \\ \mathrm{u}, \mathrm{V} & \text { Velocity components } \\ \vec{u} & \text { Velocity vector } \\ u & \\ U_{\infty} & \text { Free stream velocity } \\ l & \text { Turbulence length } \\ \mathrm{k} & \text { Turbulence kinetic energy } \\ \varepsilon & \text { Turbulence dissipation } \\ \mathrm{St} & \text { Strouhal number }\end{array}$

$\begin{array}{ll}\mathrm{Cp} & \text { Pressure coefficient } \\ \omega_{\mathrm{w}} & \text { Wall vorticity } \\ \mathrm{C}_{\mathrm{D}} & \text { Drag coefficient } \\ \mathrm{C}_{\mathrm{DP}} & \text { Drag coefficient due to pressure } \\ \mathrm{C}_{\mathrm{DV}} & \text { Drag coefficient due to viscosity } \\ \mathrm{P}_{\mathrm{w}} & \text { Dimensionless wall pressure } \\ \mathrm{Re} & \text { Reynolds number } \\ \mathrm{T} & \text { The period of vortex shedding. } \\ f & \text { Frequency of the vortex shedding } \\ I & \text { Turbulence Intensity } \\ \mathrm{C} \mu & \text { Empirical constant } \\ \omega & \text { Specified dissipation rate }\end{array}$

\section{Introduction}

Flow around a circular cylinder is a fundamental fluid mechanics problem of practical importance. It has potential relevance to a large number of practical applications such as submarines, off shore structures, bridge piers, pipelines etc. The laminar and turbulent unsteady viscous flow behind a circular cylinder has been the subject of numerous experimental and numerical studies, especially from the hydrodynamics point of view. According to the observation of Sumer (1997); the flow field over the circular cylinder is symmetric at low values of Reynolds number. As the Reynolds number increases, flow begins to separate 
behind the cylinder causing vortex shedding which is an unsteady phenomenon. For the $40<\operatorname{Re}<200$, there is a laminar vortex shedding in the wake of the cylinder. The laminar wake transient to turbulence in the region of $\operatorname{Re}=200$ to 300. In the subcritical region $300<\operatorname{Re}<3 \times 10^{5}$ the wake behind the circular cylinder becomes completely turbulent and a laminar boundary layer separation occurs. The unsteady flow was first studied by Payne (1958) for Reynolds number equal to 40 \& 100 . The numerical study and physical analysis of the pressure and velocity fields in the near wake of a circular cylinder has been investigated by Braza et al (1986). Tuann \& Olson (1978), Martinez \& Minh (1978), Loc (1980), Coutanceau \& Defaye (1991) and recently Lakshmipathy (2004) and Reichl et al. (2005) have investigated this problem for different Reynolds numbers. Braza et al (1990) predicted the large-scale transition features in the wake of a circular cylinder for $2000<\mathrm{Re}<10000$. The turbulent flow over cylinder was also investigated by Rai \& Moin (1993) and Mittal (1995) for high Reynolds number. The common points of interest of this work are the development of the primary unsteady wake behind the circular cylinder and the evaluation of the drag coefficient and the separation angle with time.

Most of the experimental studies investigated the steady and unsteady behaviors of the alternating vortices in the wake. The work of Tritton (1971), Lourenco \& Shih (1993), and Anderson (1995) should be mentioned. Besides these theoretical and numerical investigations, some experimental visualizations have been described by Honji \& Taneda (1969), Beaudan \& Moin (1994) and Coutanceau \& Bouard (1977).

All of the above numerical studies have some common characteristics: they solve the unsteady NavierStokes equation in two dimensional Helmholdtz (vorticity \& stream function) formulation; they described the relevant flow by the global parameters such as Strouhal number as a main feature of the unsteady wake, drag and lift coefficients in the wall region; nevertheless, poor analysis is provided for the near wake characteristics. The main goal of the present study is consequently to (i) visualize the laminar and turbulent unsteady flow field for different Reynolds numbers; (ii) check the capability of different turbulent models for the simulation the unsteady flow over circular cylinder. (ii) compute drag coefficients along the surfaces of the cylinder for different Reynolds numbers; (iii) plot contours of stream function, velocity vectors and static pressure to visualize vortex shedding at different time for $\mathrm{Re}=100,1000 \& 3900$. Numerical results presented here are compared with experimental measurements and other numerical results and the agreements are found satisfactory.

\section{Physical Model}

The flow field around the cylinder is modeled in two dimensions with the axes of the cylinder perpendicular to the direction of flow. The cylinder is modeled as a circle and a square flow domain is created surrounding the cylinder. The flow from left to right with the cylinder of diameter, $\mathrm{d}$ submersed in an incompressible fluid is considered. The computational domain consists of an upstream 23 times the radius to downstream 40 times the radius and the width of the domain is 50 times the radius of the cylinder. The problem setup together with the important dimensions is shown in Fig.1.

A typical rectangular mesh used for simulation of this study. This particular mesh has approximately 15659 nodes, 30924 faces and 15380 quadrilateral cells with considerable mesh concentration both around the cylinder and in the wake. To facilitate meshing, a square with side length of three times diameter of the cylinder is created around the cylinder.

\section{Mathematical Model}

\subsection{Governing Equations}

The governing equations of the unsteady flow of an incompressible viscous fluid past a circular cylinder are the classical continuity and Navier- Stokes equations. They are written in the following form:

$$
\operatorname{div}(\vec{u})=0
$$

Numerical Investigation of Unsteady Flow Past a Circular Cylinder using 2-D Finite Volume Method 


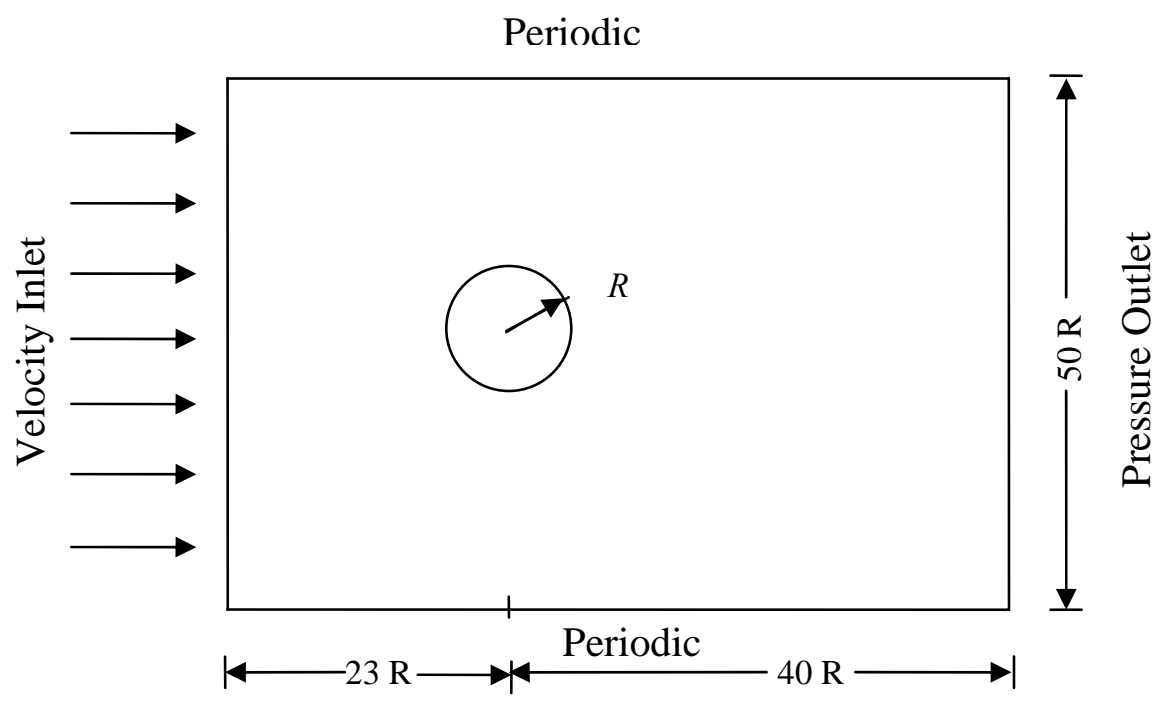

Fig.1: Schematic diagram of the flow field around circular cylinder

$\frac{\partial(u)}{\partial t}+\operatorname{div}(u \vec{u})=\frac{1}{\rho} \operatorname{div}(\Gamma \nabla u)$

Equation (2) has the rate of change term and the convective term in the left hand side and the diffusion term ( $\Gamma=$ diffusion coefficient) on the right hand side. The equation is used as the starting point for computational procedure in finite volume method. The key step of the finite volume method (Versteeg \& Malalsekera,1995) is the integration of Equation (2) over a two dimensional control volume CV yielding

$$
\int_{C V} \frac{\partial(u)}{\partial t} d v+\int_{C V} d i v(u \vec{u}) d v=\frac{1}{\rho} \int_{C V} \operatorname{div}(\Gamma \nabla u) d v
$$

The volume integrals in the $2^{\text {nd }}$ term on the left hand side, the convective term and in the right hand side the diffusion term can be re- written as integrals over the entire boundary surface of the control volume by using Gauss divergence theorem. Then Equation (3) becomes

$\frac{\partial}{\partial t}\left(\int_{C V}(u) d v\right)+\int_{A} n \cdot(u \vec{u}) d A=\frac{1}{\rho} \int_{A} n \cdot(\Gamma \nabla u) d A$

In the steady state case, the rate of change term is equal to zero. This leads to the integrate form of the steady transport equation

$$
\int_{A} n \cdot(u U) d A=\frac{1}{\rho} \int_{A} n \cdot(\Gamma \nabla u) d A
$$

In time dependent (unsteady) case, it is also necessary to integrate with respect to time $t$ over a small time interval $\Delta t$ from (say) $\mathrm{t}$ to $t+\Delta t$. This yields the most general integral form of the transport equation.

$$
\int_{\Delta t} \frac{\partial}{\partial t}\left(\int_{C V}(u) d v\right) d t+\iint_{\Delta t A} n \cdot(u \vec{u}) d A d t=\frac{1}{\rho} \int_{\Delta t} \int_{A} n .(\Gamma \nabla u) d A d t
$$


In finite volume method, flow domain is divided into a number of control volumes or cells. Discritized form of Eq. (6) must be set up at a nodal point placed within each control volume in order to solve the problem. For control volumes adjacent to the domain boundaries, the general equation is modified to incorporate boundary conditions. The resulting system of linear algebraic equations is then solved to obtain the velocity and pressure distribution at each nodal point. Finally, drag and lift coefficients are computed as follows:

$$
\begin{aligned}
& C_{D}=\frac{D}{0.5 \rho U^{2}{ }_{\infty} d} \quad C_{D P}=\int_{0}^{2 \pi} P_{w} \cos d x C_{D V}=\frac{2}{\mathrm{Re}} \int_{0}^{2 \pi} \omega_{w} \sin x d x \\
& C_{L}=\frac{L}{0.5 \rho U^{2}{ }_{\infty} d} \quad C_{L p}=\int_{0}^{2 \pi} P_{w} \sin x d x \quad C_{L V}=\frac{2}{\operatorname{Re}} \int_{0}^{2 \pi} \omega_{w} \cos x x d x
\end{aligned}
$$

Where, D \& L represent drag and lift force.

Also, the pressure coefficient is defined as: $C_{P}=\left(P-P_{\infty}\right) / \frac{1}{2} \rho U^{2} d$.

Where, the subscripts $\mathrm{P}$ and $\mathrm{V}$ respectively represent the contributions of the pressure and viscous force. $\mathrm{P}_{\mathrm{w}}$ is the dimensionless wall pressure and $\omega_{\mathrm{w}}$ is the dimensionless wall vorticity defined as $\omega_{w}=\omega R / U_{\infty}$, (here $R=0.5$ ). The dimensionless Reynolds number is given by $\operatorname{Re}=d U_{\infty} \rho / \mu$

Also, the dimensionless Strouhal number is expressed as $S t=f d / U_{\infty}$

Where, $f$ is the frequency of the vortex shedding $(=1 / T)$, $d$ is the diameter of the cylinder and $U_{\infty}$ the free stream velocity.

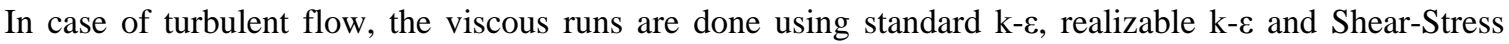
Transport (SST) k- $\omega$ model (Lakshmipathy, 2004).

The standard k- $\varepsilon$ model is a semi-empirical model based on model transport equations for the turbulence kinetic energy $(\mathrm{k})$ and its dissipation rate $(\varepsilon)$. The model transport equation for $\mathrm{k}$ is derived from the exact equation, while the model transport equation for $\varepsilon$ was obtained using physical reasoning and bears little resemblance to its mathematically exact counterpart. In the derivation of the k- $\varepsilon$ model, it was assumed that the flow is fully turbulent, and the effects of molecular viscosity are negligible. The standard k- $\varepsilon$ model is therefore valid only for fully turbulent flows.

On the other hand the term "realizable" means that the model satisfies certain mathematical constraints on the normal stresses, consistent with the physics of turbulent flows. The most straightforward way to ensure the realizability is to make $\mathrm{C} \mu$ variable by sensitizing it to the mean flow (mean deformation) and the turbulence $(k, \varepsilon)$. The turbulence kinetic energy $k$ is given by

$k=\frac{3}{2}\left(U_{\text {avg }} I\right)^{2}$

Where, $U_{\text {avg }}$ is the mean flow velocity and $I$ is the turbulence intensity defined by

$I=0.16(\mathrm{Re})^{-\frac{1}{8}}$

Turbulence length can be written as $l=0.07 d$ and turbulence dissipation rate $\varepsilon$ as 


$$
\varepsilon=C_{\mu} \frac{3}{4} \frac{k^{\frac{3}{2}}}{l}
$$

Where, $C_{\mu}$ is an empirical constant specified in the turbulent model (approximately 0.09).

In this study, another turbulent model is used to simulate the turbulent flow named shear-stress transport (SST) k- $\omega$ model. It is so named because the definition of the turbulent viscosity is modified to account for the transport of the principal turbulent shear stress. It is such a feature that gives the SST k- $\omega$ model an advantage in terms of performance over both the standard $k-\varepsilon$ and realizable $k-\varepsilon$ model. Other modification includes the addition of a cross-diffusion term in the $\omega$-equation and a blending function to ensure that the model equations behave appropriately in both the near-wall and far-field zones. In SST k- $\omega$ model the specific dissipation rate $\omega$ can be found by

$$
\omega=\frac{k^{\frac{1}{2}}}{C_{\mu}^{\frac{1}{4}} l}
$$

The control volume technique consists of integrating the governing equations about each control volume, yielding discrete equations that conserve each quantity on a control volume basis. The coupling of pressure and velocity is done using PISO algorithm (Versteeg \& Malalsekera ,1995).

\subsection{Boundary Condition}

The wall boundary conditions used in this steady are those of impermeability and non-slip condition, i.e., $\mathrm{u}$ $=0, \mathrm{v}=0$

In the physical domain the flow is not confined. Nevertheless, a fictitious external rectangle boundary is needed at a large distance from the cylinder (Fig.1) in order to solve the governing equations numerically. Uniform free stream condition with velocity $U_{\infty}=1.0 \mathrm{~m} / \mathrm{s}$ are applied at the inlet boundary. The periodic condition is considered at the lateral boundaries. Also the flow exit is treated as a pressure outlet.

\section{Results and Discussion}

\subsection{The vortex shedding:}

\subsubsection{The Laminar Flow $(\mathrm{Re}=100)$}

Experimental result shows that, for Reynolds number less than 40 the flow over the circular cylinder reaches the steady state within 15 second and two symmetric vortices are formed behind the cylinder ( Braza et al, 1986). As Reynolds number becomes higher than 40 the flow reports a loss of symmetry in the wake and alternating eddies are formed and convected in the wake. This generates the alternating separation of vortices, which are convected and diffused away from the cylinder, forming the well-known Karman vortex streets. Such destabilizing effects always occur during any physical experiment on the flow around a circular cylinder. The Strouhal number (St) is found to be 0.164 for $\mathrm{Re}=100$. This result agrees well with the experimental value (0.164 0.165) reported by Tritton (1959).

The results of unsteady laminar flow for $\mathrm{Re}=100$ are presented in Figs. 2-4. The instantaneous stream lines for $\mathrm{Re}=100$ are shown in Fig. 2 at the four phases during one cycle of vortex shedding. In Fig. 3 \& Fig. 4 the directions of the velocity vectors and corresponding contours of pressure are plotted respectively at those phases for the same Reynolds number. The alternating formation, convection and diffusion of the vortices are clearly shown. 


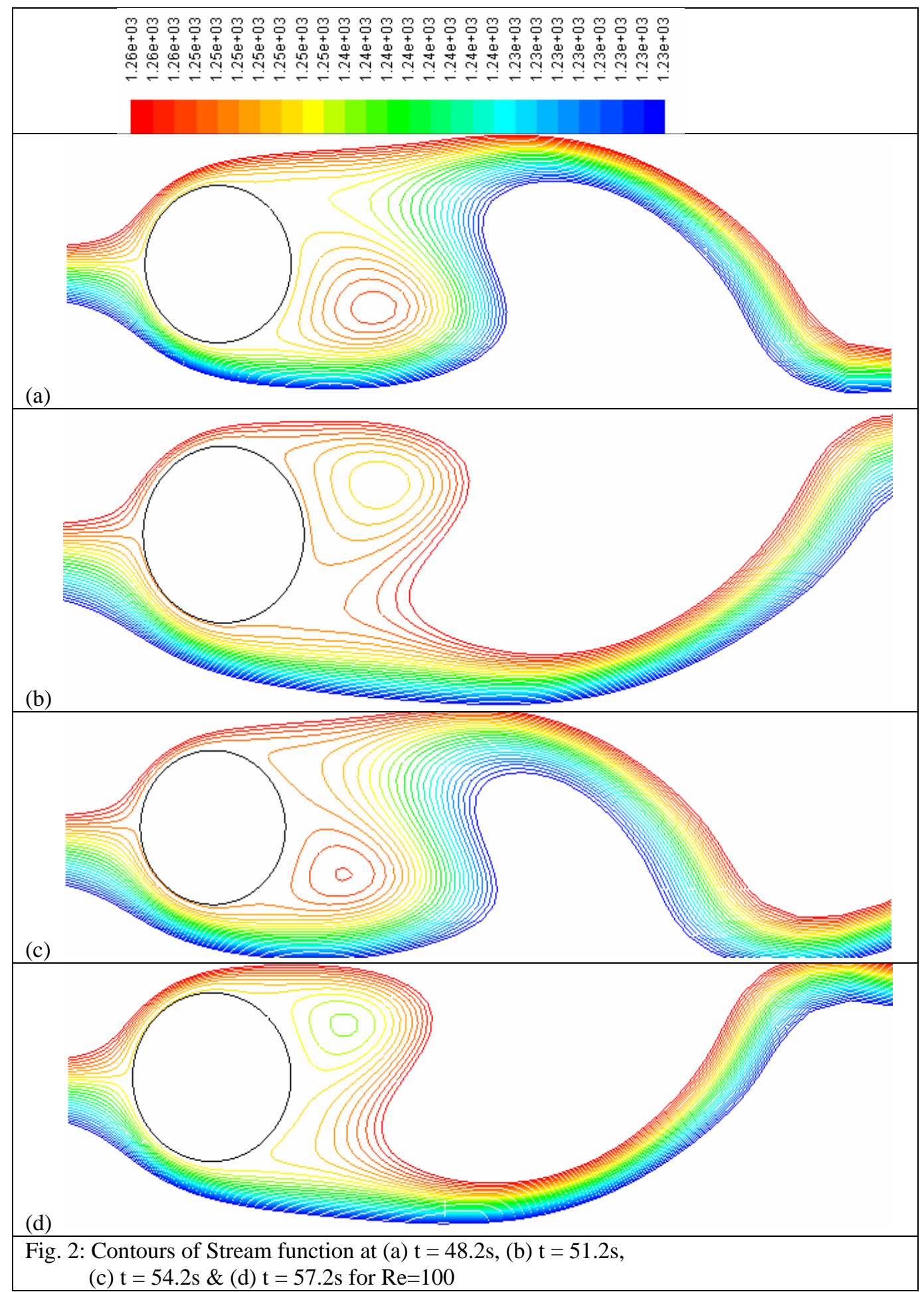

Numerical Investigation of Unsteady Flow Past a Circular Cylinder using 2-D Finite Volume Method 

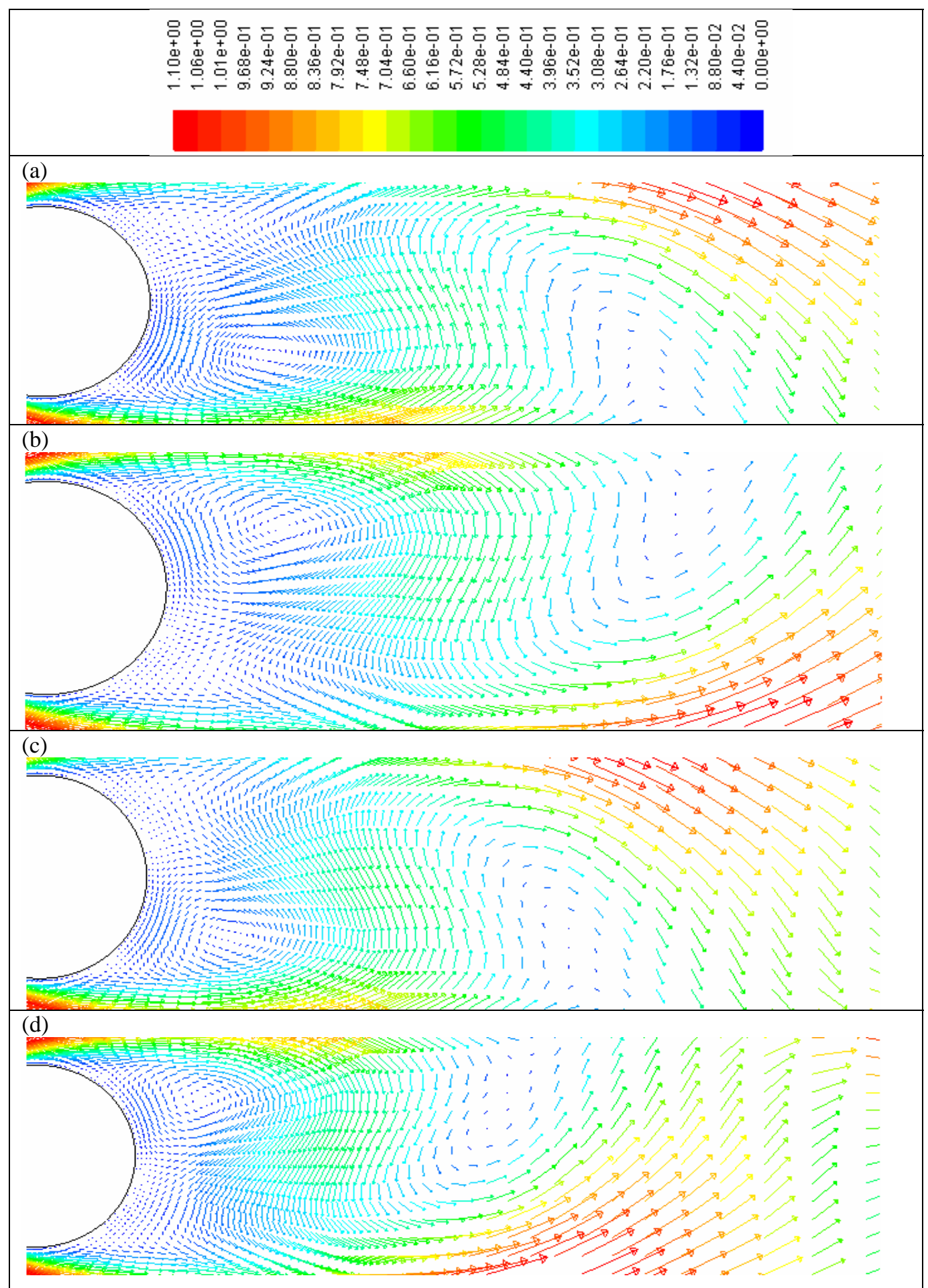

Fig. 3: Contours of Velocity vectors at (a) $t=48.2 s$, (b) $t=51.2 s$, (c) $t=54.2 s \&$ (d) $t=57.2 s$ for $\mathrm{Re}=100$ 


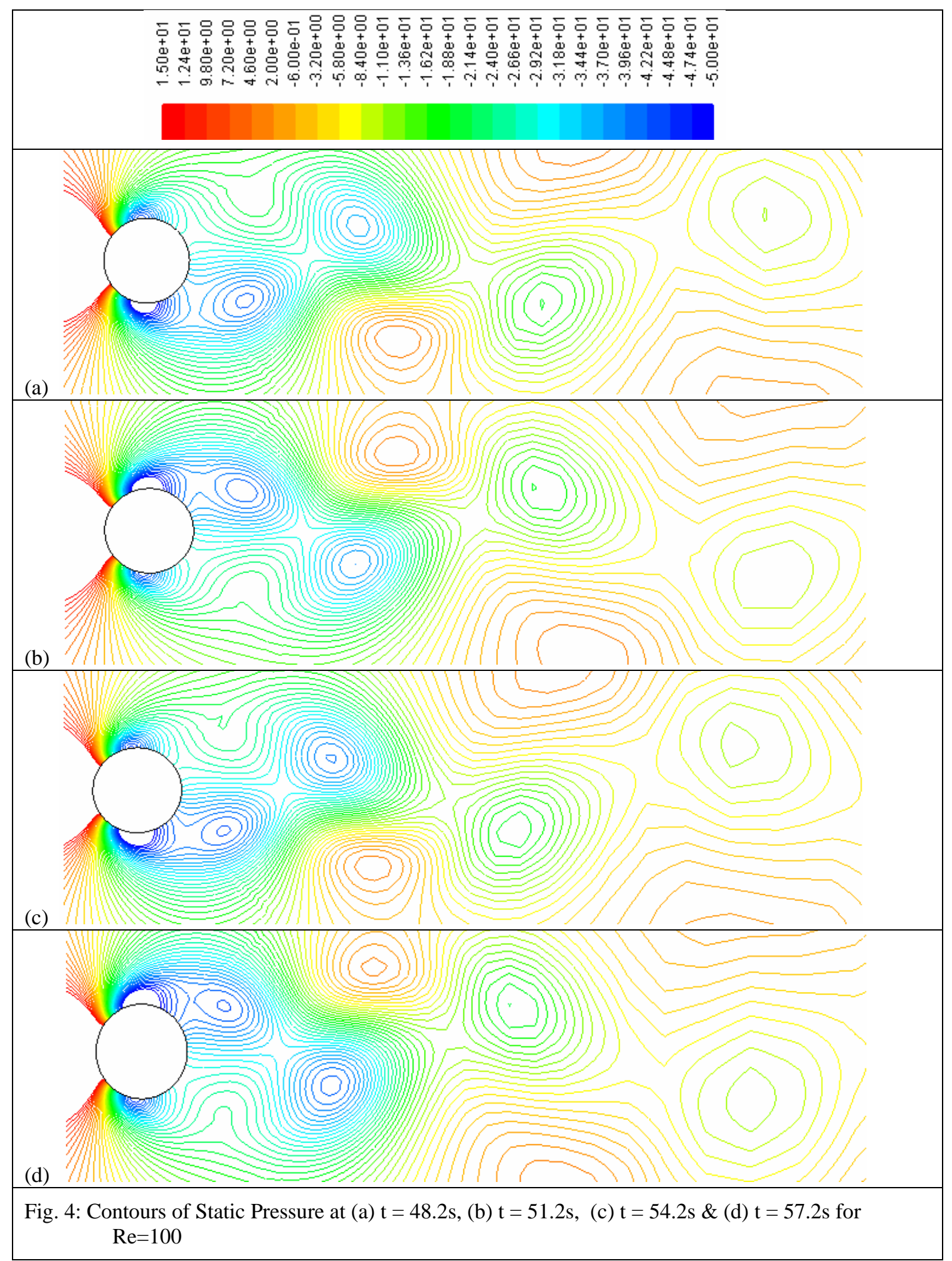




\subsubsection{The Turbulent Flow (Re $=1000$ and 3900)}

The experimental work of Roshko (1954) locates the beginning of the laminar-to-turbulent transition at Reynolds numbers $200-300$. Beyond this Reynolds number but less than $3 \times 10^{5}$ the wake of the cylinder is completely turbulent and the boundary layer separation is laminar (Sumer, 1997). It is known that the flow around the cylinder is two dimensional only when $\operatorname{Re}<200$. For Larger Reynolds number, the vortex shedding occurs in cells and therefore the flow is generally simulated in 3-D. But the 3-D simulation is very much complicated and treated as a numerically costly procedure. Therefore, the turbulent flow over a circular cylinder for Reynolds numbers 1000 \& 3900 is investigated here using 2-D finite volume method.

In this research, the turbulent flow for both Reynolds numbers is simulated using three turbulence models namely, Standard $\mathrm{k}-\varepsilon$, Realizable $\mathrm{k}-\varepsilon$ and Shear-Stress Turbulence (SST) k- $\omega$ turbulence model. The vortex shedding is observed and the drag \& lift forces is also calculated using these methods.

For unsteady case, time interval have been chosen small enough to capture the vortex shedding. The easiest way to set up the right time step size is one that ensures the maximum Courant number very near to unity. To avoid running the case for very long time, a small perturbation has been introduced in the domain. This perturbation is generally in the form of very small velocity in perpendicular direction to the flow direction. This assists the solver to start vortex shedding. Since there is nothing to sustain this perturbation in the domain for very long time, it dies out and does not affect the calculation of $\mathrm{C}_{l}$ and $\mathrm{C}_{\mathrm{d}}$. The dimensionless frequency of vortex shedding is the Strouhal numbers found by different methods are shown in Fig.5.

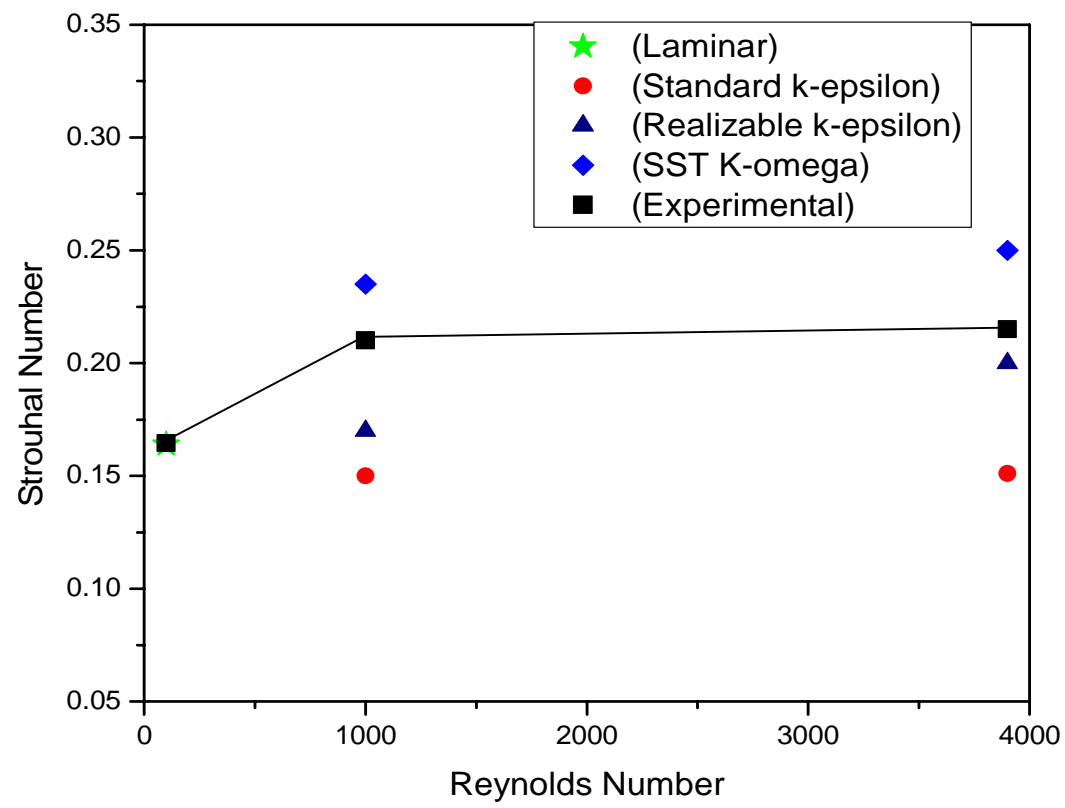

Fig.-5: Strouhal Number Vs Reynolds Number

The vortex shedding is visualized by means of the contours of stream function, velocity vectors and static pressure. Fig. 6 show the stream function during one cycle of lift coefficient for $R e=1000$. At the same time the velocity vector and pressure fields are also shown in Fig. 7. The alternating vortex shedding clearly observed in these figures. Fig. 8 shows the stream function and Fig. 9 shows the velocity vector $\&$ pressure field during one cycle of lift coefficient for $\mathrm{Re}=3900$. The alternating formation, convection and diffusion of the vortices are clearly shown again. 
The results in this section show that the vortex shedding is an intrinsic phenomenon of the flow, well predicted by the solution of the Navier- Stokes equations. The vortex shedding is generated by a loss of symmetry of the two dimensional symmetric structures in the wake of the circular cylinder.

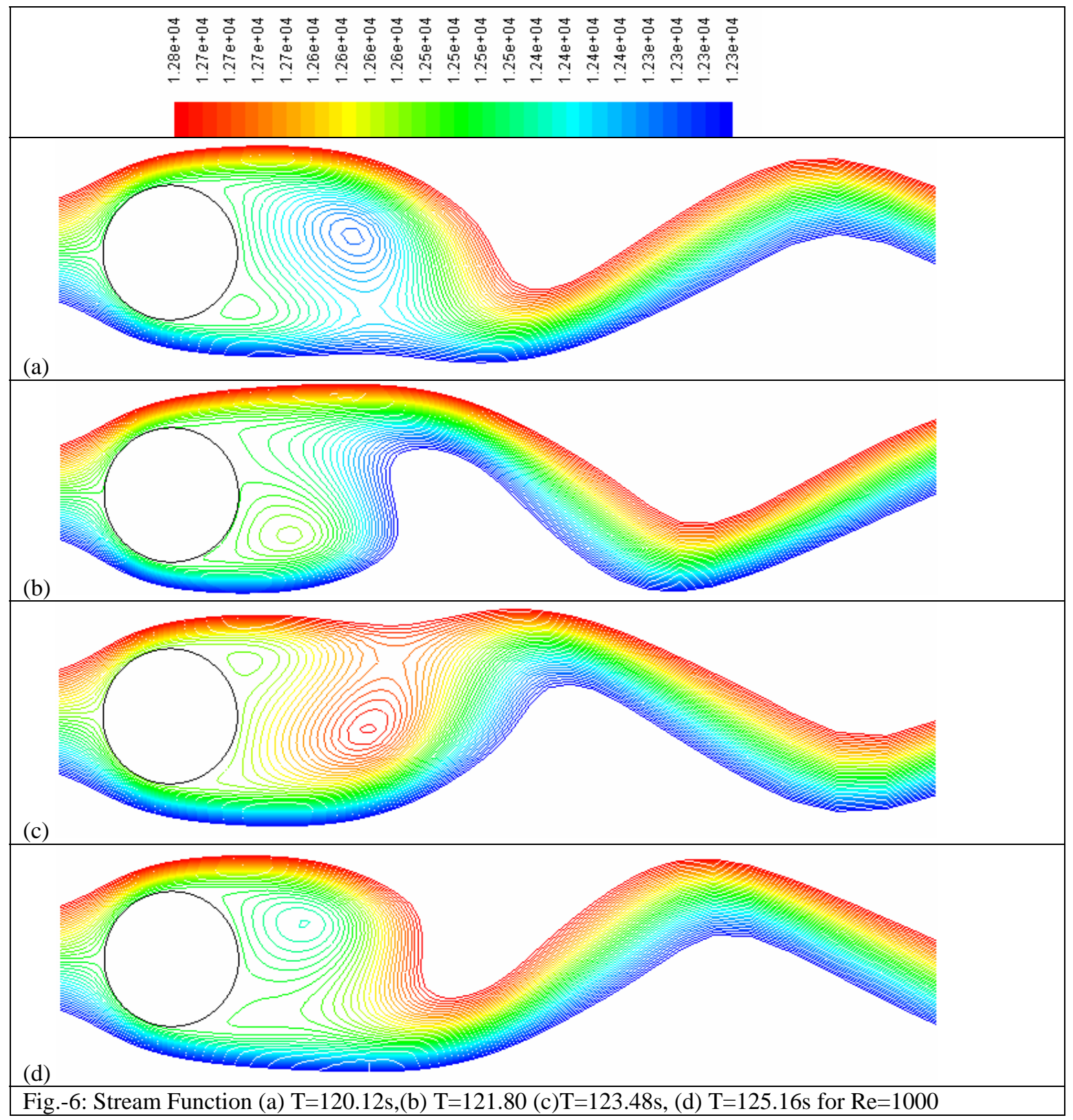



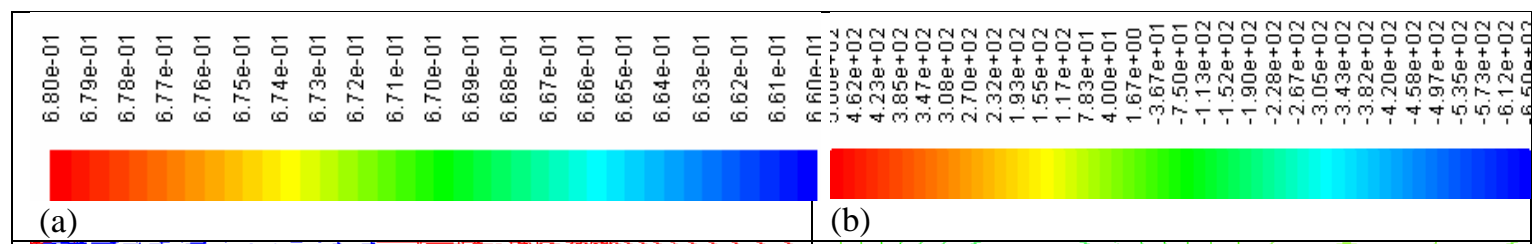

(b)

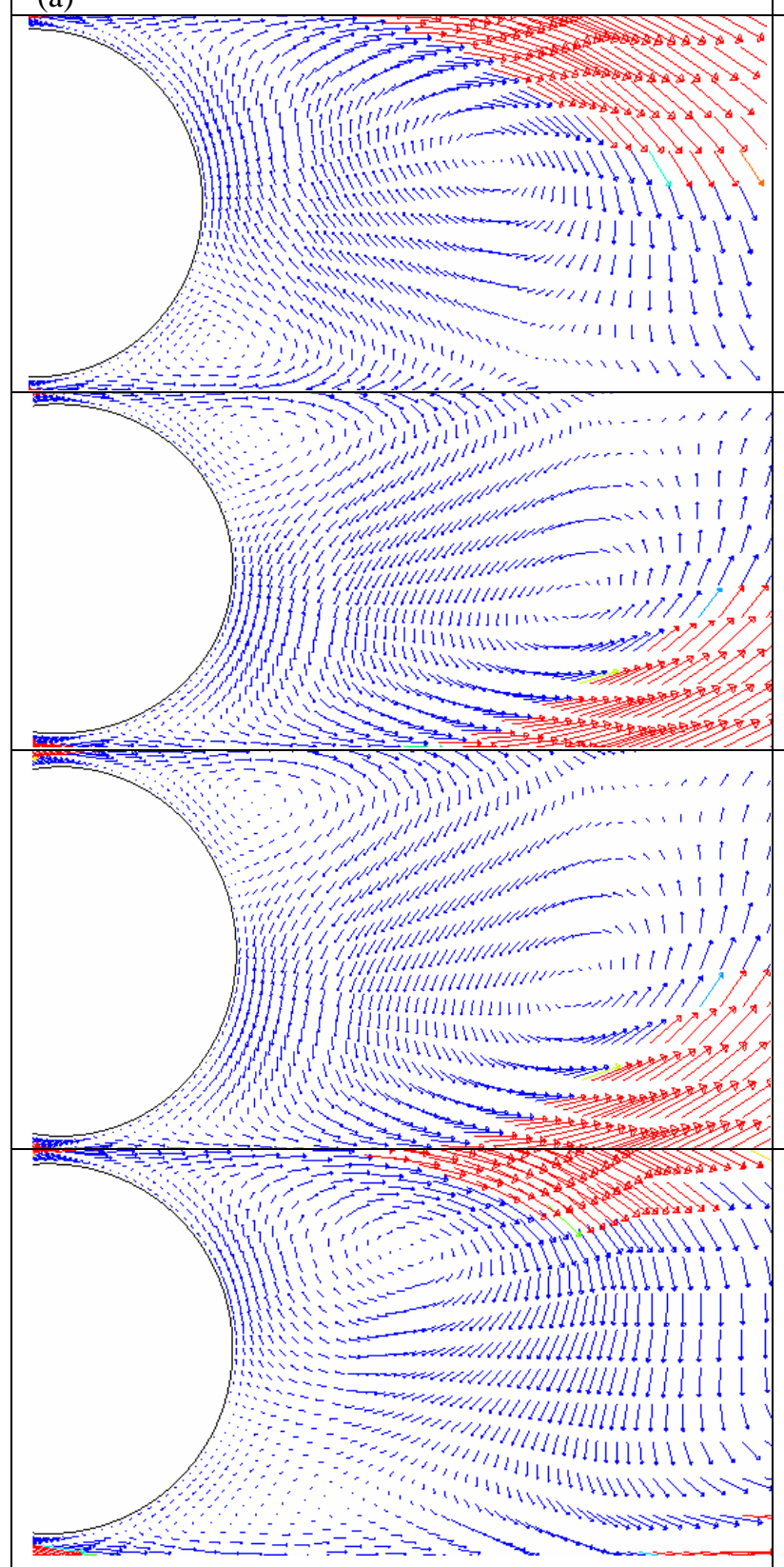

(b)

Fig.-7: (a) Velocity Vector (b) Static Pressure at T=120.12s, T=121.80 T=123.48s, T=125.16s for $\operatorname{Re}=1000$ 


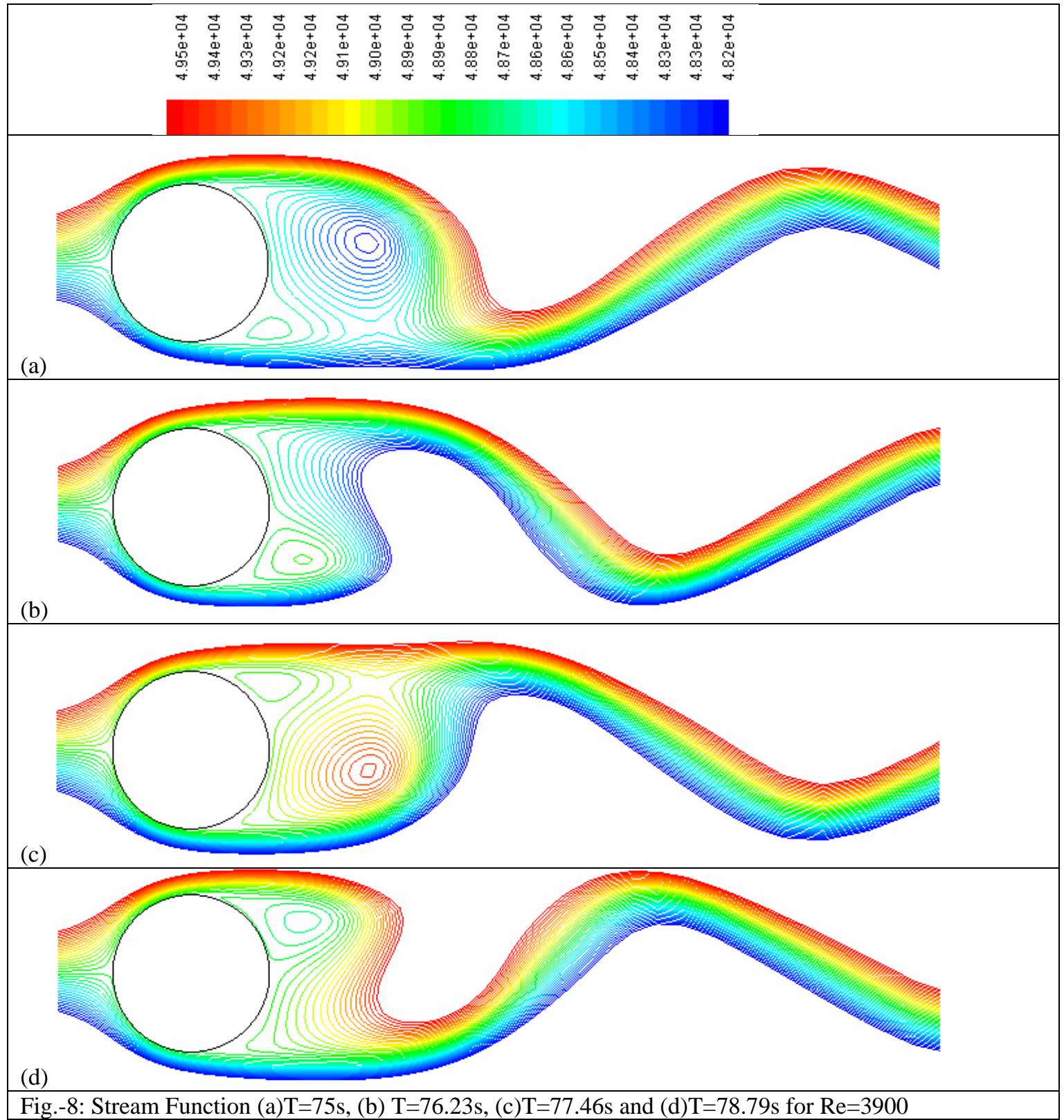

\subsection{The Forces:}

The drag force is a result of the convective motion of the cylinder through the fluid. Because of this motion and of the non-slip condition of the wall, a tangential velocity gradient is created in the direction normal to the wall. The mean value of the drag coefficient calculated by the present method for different Reynolds numbers is very close to the experimental results of Tritton (1959), Tritton (1971) and Anderson (2005). The total drag is also due to two effects: the pressure field $\left(\mathrm{C}_{\mathrm{Dp}}\right)$ and the viscous forces $\left(C_{D v}\right)$ calculated by the Eqs. $(7-8)$. For $R e=100$ the mean value of the pressure drag, $C_{D p}$ is 0.917 , which corresponds to $75 \%$ of the total drag. This value is closer to the experimental result (0.995) of 
Roshko (1954) than that (1.02) of Braza et al.(1986). Numerical values of the components of total drag coefficient are shown in Table 1.
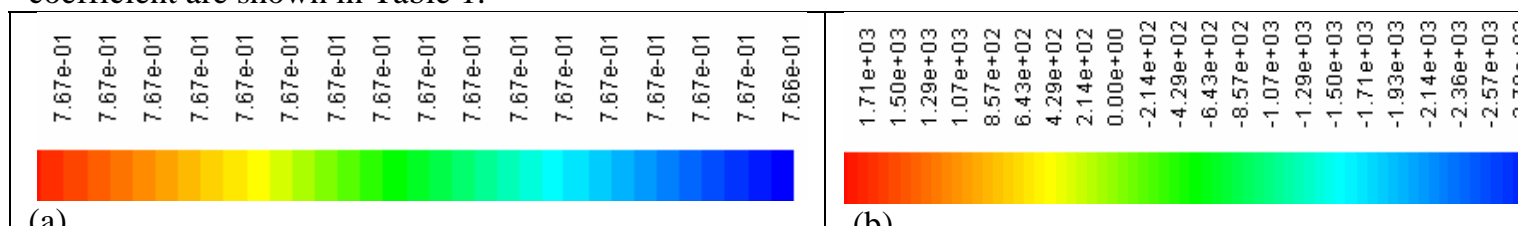

(a)

(b)

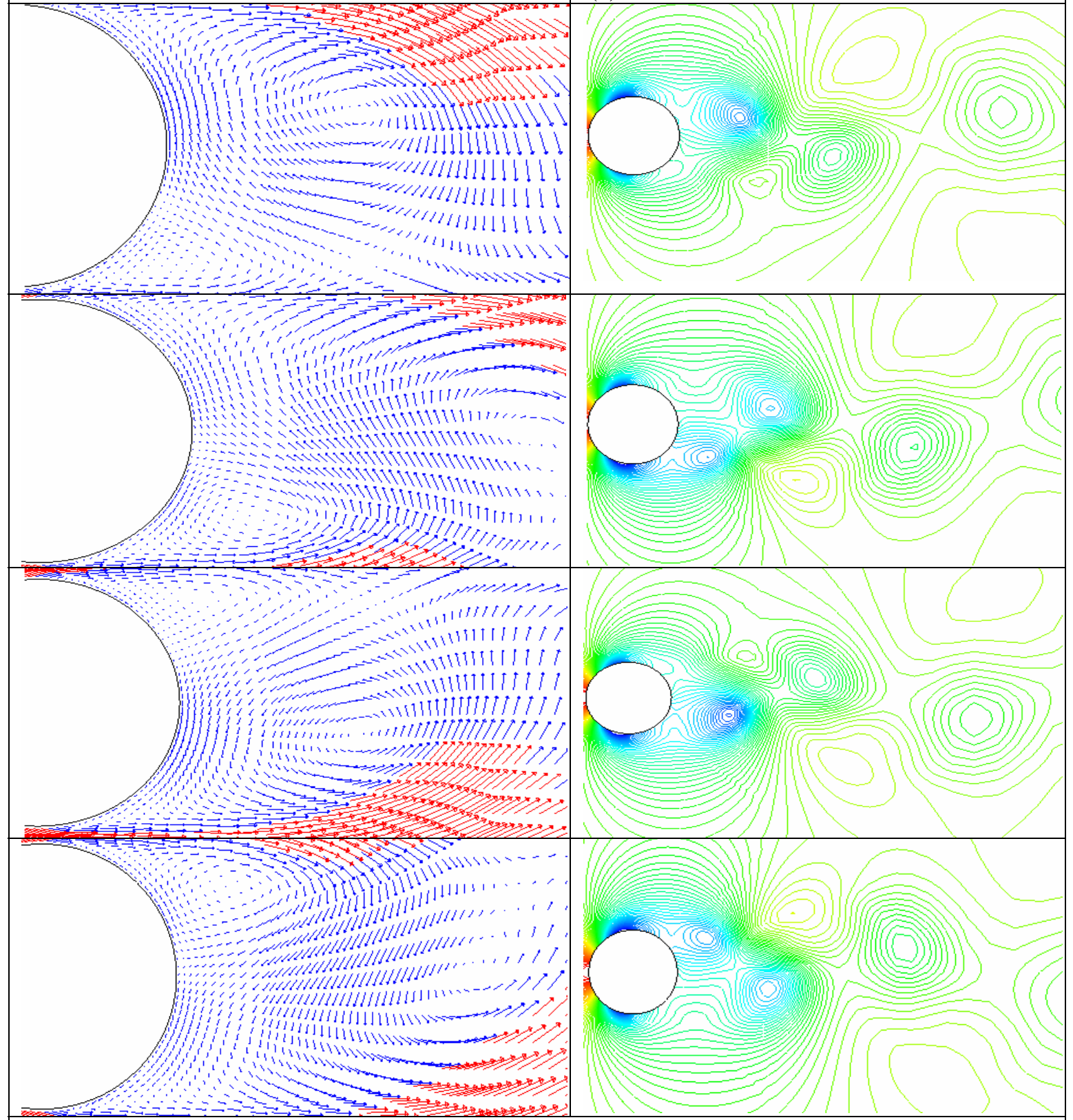

Fig.-9: (a) Velocity Vector (b) Static Pressure at T=75s, T=76.23s, T=77.46s and T=78.79s for Re=3900

Numerical Investigation of Unsteady Flow Past a Circular Cylinder using 2-D Finite Volume Method 
The pressure and drag coefficients of the turbulent flow at $\mathrm{Re}=1000 \& 3900$ have also been calculated using three turbulent models. The predicted $\mathrm{C}_{\mathrm{d}}$ values by standard k-epsilon model are quite closely to the experimental values shown in Table 2. But for the visualization of vortex shedding the Realizable k-epsilon turbulence model is more effective, as this model captures the separating flow better than standard kepsilon model.

On the other hand, the SST k-omega model is much more recommendable for high Reynolds numbers. The global periodic character of the flow is found to be essentially the same as the Reynolds number increases. For the Reynolds numbers of 1000 and 3900 it should be recalled that the unsteady lift and drag coefficient oscillates periodically. Fig. 10 shows the lift and drag coefficient for $\mathrm{Re}=1000$. The drag coefficients as a function of different Reynolds numbers are compared with experimental and other numerical results in Fig. 11 and in Table 2. In most of the cases, the computed results show better agreement with experimental data compared to other predicted values.
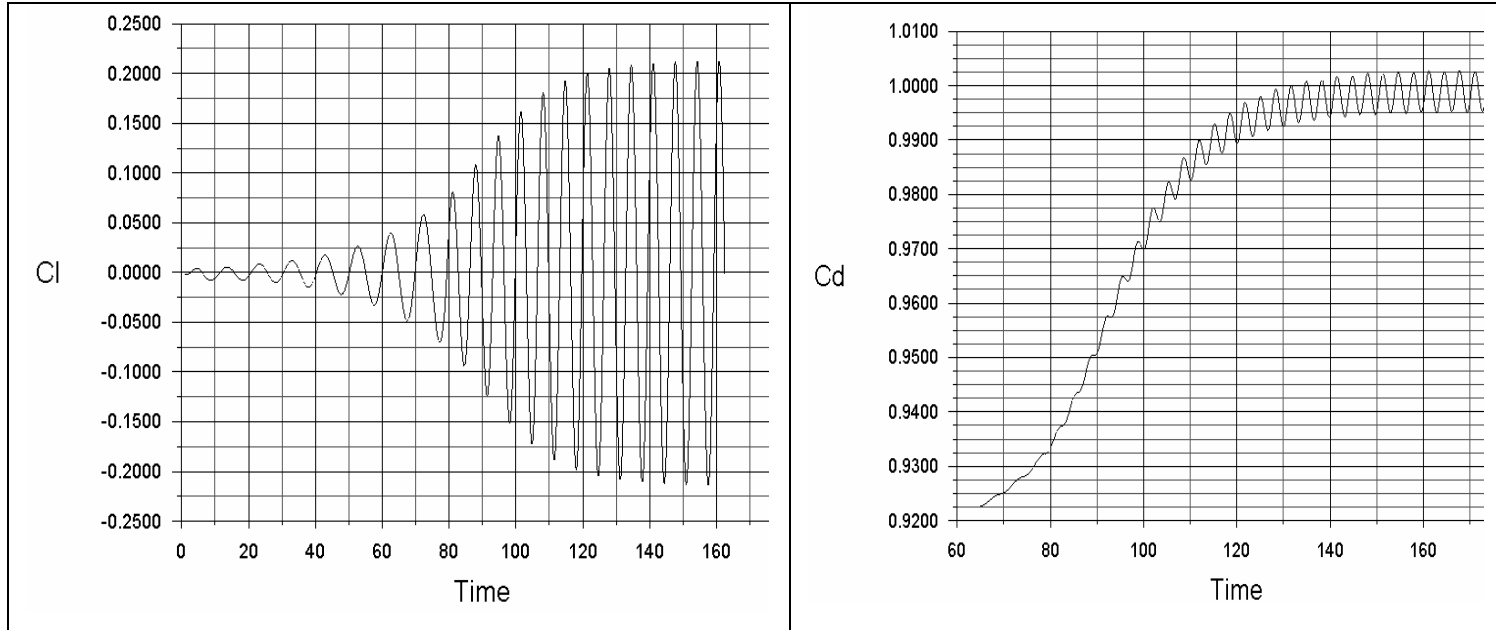

Fig.-10: Time History of (a) Lift coefficient and (b) Drag coefficient at $\mathrm{Re}=1000$

Table 1: Components of Total Drag Coefficient.

\begin{tabular}{|c|c|c|c|}
\hline Re & Pressure Coefficient & Viscous coefficient & Total Drag Coefficient \\
\hline 100 & 0.917 & 0.329 & 1.245 \\
\hline 1000 & 0.876 & 0.119 & 0.995 \\
\hline 3900 & 0.877 & 0.12 & 0.997 \\
\hline
\end{tabular}

\section{Conclusion}

The complex problem of the unsteady laminar and turbulent flow around a circular cylinder at Reynolds numbers of 100, 1000 and 3900 is studied using 2-D finite volume method. A second-order-accurate numerical scheme is used, based upon a velocity-pressure formulation and conservative schemes. Three different turbulent models are used to tackle the complex behavior of turbulent flow. From this study it is found that the two-dimensional finite volume method computes hydrodynamic forces and captures vortex shedding very well. Even at high Reynolds number, the method is very much applicable without loss of 
accuracy. It is also observed that standard k-epsilon model computes drag coefficients accurately, where the realizable k-epsilon turbulence model is more effective for visualization of vortex shedding. The SST komega model is much more recommendable for high Reynolds numbers. Most of the results presented in this research are compared with experimental data and are in better agreement with respect to other numerical results. In this research, 2-D method is found very prospective one for turbulent flow due to its faster convergence. However, the method is restricted here only at two Reynolds numbers (1000 \& 3900) to analyze turbulent flow around circular cylinder; more studies are required to make it applicable at higher Reynolds numbers.

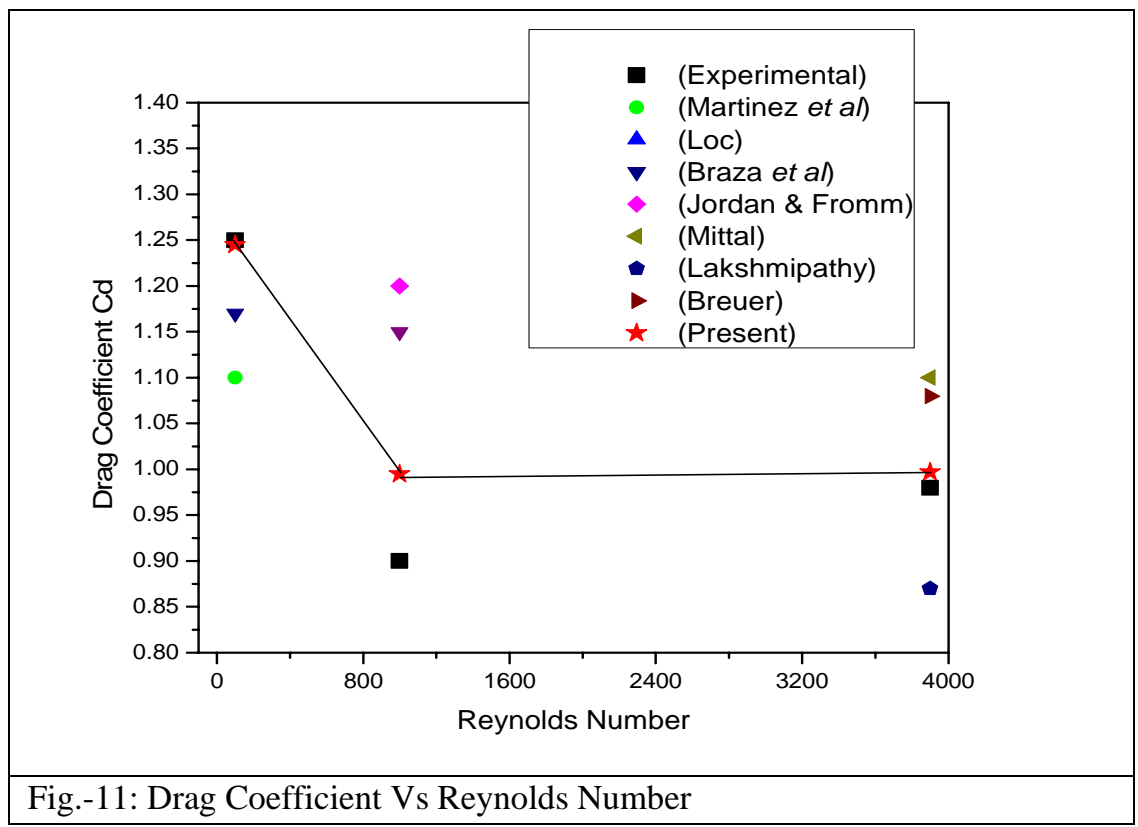

Table2: Drag coefficient for different Reynolds numbers with experimental and some numerical results.

\begin{tabular}{|l|c|c|c|}
\hline \multicolumn{1}{|c|}{ Re } & $\mathbf{1 0 0}$ & $\mathbf{1 0 0 0}$ & $\mathbf{3 9 0 0}$ \\
\hline Experimental & $\begin{array}{c}1.24-1.26 \\
\text { (Tritton,1959) }\end{array}$ & $\begin{array}{c}0.9 \\
\text { (Anderson, 2005) }\end{array}$ & $\begin{array}{c}0.98+/-0.05 \\
\text { Lourenco \& Shih (1993) }\end{array}$ \\
\hline Tuann \& Olson (1978) & 1.25 & -- & -- \\
\hline Martinez et al. (1978) & 1.1 & -- & -- \\
\hline Loc (1980) & 1.15 & -- & -- \\
\hline Braza et al. (1986) & 1.17 & 1.15 & -- \\
\hline Jordon \& Fromm(1972) & -- & 1.2 & 1.74 \\
\hline Beaudan \& Moin (1994) & -- & -- & 0.87 \\
\hline Lakshmipathy & -- & -- & 1.08 \\
\hline Breuer (1998) & -- & -- & 0.997 \\
\hline Present & 1.245 & 0.995 & - \\
\hline
\end{tabular}




\section{References}

Anderson, J.D. (2005): Fundamentals of Aerodynamics, $4^{\text {th }}$ Ed., Mc Grow-Hill, Columbus, USA.

Braza, M., Chassaing, P. and Minh, H. H.(1986): The Numerical Study and Physical Analysis of the Pressure and Velocity Fields in the Near Wake of a Circular Cylinder, J. Fluid Mech., 165, 79.

Braza, M., Chassaing, P. and Minh,H. H.(1990): Prediction of Large-Scale Transition features in the wake of a Circular Cylinder, Phys. Fluids. A2 (8), 1461-1471.

Beaudan, P. \& Moin, P. (1994): Numerical Experiments on the Flow Past Circular cylinders at Sub-Critical Reynolds numbers. Report No. TF-62, Thermosciences Div., Dept. of Mech. Engr., Stanford University.

Coutanceau, M. and Bouard (1977): Experimental Determination of the Main Features of the Viscous Flow in the Wake of the Circular Cylinder in Uniform Translation, J. Fluid Mech. 79, 231 \& 257.

Coutanceau, M. and Defaye, J.R. (1991): Circular Cylinder Wake Configurations- A flow Visualization Survey, Appl. Mech. Rev., 44(6).

Honji, H. and Taneda, S. (1969): Unsteady Flow Past a Circular Cylinder, J. Phys. Soc. Japan, 27, 16681677.

Lakshmipathy, S. (2004): PANS Method for Turbulence Simulation of High and Low Reynolds Number Flow Past a Circular Cylinder, M.Sc. Thesis, Dept. of Aerospace Eng. Texas A. \& M. University, USA.

Loc, T. P. (1980): Numerical Analysis of Unsteady Secondary Vortices Generated by an Impulsively Started Circular Cylinder. J. Fluid Mech, 100, 111.

Lourenco, L. M. \& Shih, C. (1993): Characteristics of the Plane Turbulent Near Wake of a Circular Cylinder. A Particle Image Velocimetry Study, Private Communication.

Martinez, G. \& Ha Minh, H.(1978): In Proc. Intl. Conf. on Numerical Methods in Laminar and Turbulent Flow, Swansea, Pineridge.

Mittal, R., (1995): Large-Eddy Simulation of Floe Past a Circular Cylinder, Annual research Briefs, Center fo Turbulence Research, Stanford University.

Payne, R.B. (1958): Calculations of Unsteady Viscous Flow Past a Circular Cylinder, J. Fluid Mech. 4, 81.

Rai, M. M. \& Moin, P. (1993): Direct Numerical Simulation of Transition and Turbulence in a Spatially Evolving Boundary Layer, J. Comp. Phys. 109, No. 2, 169-192.

Reichl, P.,Hourigan, K. and Thompson, M.C. (2005): Flow Past a Circular Cylinder Close to a Free Surface, J. Fluid Mech. 533, 269-296.

Roshko, A (1954): Vortex Shedding From Circular Cylinder at Low Reynolds Number, NACA Rep. No.1191.

Sumer, B. M. (1997): Hydrodynamics Around Cylindrical Structures, World Scientific, Singapore.

Tritton, D.J. (1959): Experiments on the Flow Around a Circular Cylinder at Low Reynolds Number, J. Fluid Mech. 6, 547.

Tritton, D. J.(1971): A Note on Vortex Streets behind Circular Cylinders, J. Fluid Mech. 45,203.

Tuann, S. Y.\& Olson, M. D. (1978): Numerical Studies of the Flow Around a Circular Cylinder by Finite Element Method, Computers and Fluids, 6, 219

Versteeg,H.K \& Malalasekera, W. (1995): An Introduction to Computational Fluid Dynamics, Longman Scientific \& Technical, England. 